\title{
Le Corbusier et le Japon, traduit par E. Peschard-Erlih, sous la direction de G. Monnier
}

\author{
Minami Asuka
}

\section{(2) OpenEdition \\ 12 Journals}

Édition électronique

URL : https://journals.openedition.org/cipango/434

DOI : $10.4000 /$ cipango.434

ISSN : 2260-7706

\section{Éditeur}

INALCO

\section{Édition imprimée}

Date de publication : 1 janvier 2008

Pagination : 252-257

ISBN : 978-2-85831-177-4

ISSN : $1164-5857$

Référence électronique

Minami Asuka, «L Corbusier et le JAPON, TRAduit PAR E. Peschard-erlih, SOUS LA DiRection de G. MONNIER », Cipango [En ligne], 15 | 2008, document 5, mis en ligne le 13 novembre 2011, consulté le 30 juin 2021. URL : http://journals.openedition.org/cipango/434; DOI : https://doi.org/10.4000/ cipango.434

Ce document a été généré automatiquement le 30 juin 2021.

\section{(c) (1) (9)}

Cipango est mis à disposition selon les termes de la Licence Creative Commons Attribution - Pas d'Utilisation Commerciale 4.0 International. 


\title{
Le Corbusier et le Japon, traduit par E. Peschard-Erlih, sous la direction de G. Monnier
}

\author{
Minami Asuka
}

\section{RÉFÉRENCE}

Le Corbusier et le Japon, traduit par E. Peschard-Erlih, sous la direction de G. Monnier, Picard, 2007, 170 p. ISBN 978-2-7084-0720-6

2007

1 Cet ouvrage se base sur la publication en japonais des actes du symposium international «Le Corbusier et le Japon » sous la direction de Takashina Shūji, Suzuki Hiroyuki, Miyake Riichi, Ōta Yasuto en 1999 aux Éditions Kajima shuppankai. Il s'est tenu en février 1997 à l'Institut architectural du Japon (AIJ) à Tōkyō dans le cadre des Septièmes Rencontres «Le Corbusier, Voyages, Rayonnement International» programmées par la Fondation Le Corbusier (Paris) et par le Comité d'organisation du symposium (Tōkyō). La version française se compose de dix des douze articles de l'ouvrage original, d'une préface de Jean-Marie Bouissou, d'une introduction de Gérard Monnier, d'annexes et de 45 illustrations.

Dans sa préface, Jean-Marie Bouissou éclaire l'évolution de l'influence de Le Corbusier à la lumière de l'histoire politique et sociale du Japon depuis l'ouverture des frontières en 1853. Le Japon éveillé s'efforça d'assimiler la science et la culture occidentales. Néanmoins, les victoires remportées lors de trois guerres (sino-japonaise, russojaponaise et la Première Guerre mondiale) rendirent nécessaire la réévaluation de la culture traditionnelle afin de définir l'identité nationale du pays. Dans le milieu architectural, un style éclectique dit teikan yōshiki (style à toiture impériale) fut élaboré et mis en oeuvre. La combinaison du toit traditionnel japonais et de la construction occidentale devait représenter l'idée de "maîtrise de la technique occidentale et 
conservation de l'esprit japonais ». Cependant, les architectes modernistes refusèrent cette chimère. Ils insistèrent sur les similitudes entre l'architecture du Mouvement moderne, notamment les œuvres corbuséennes, et l'architecture traditionnelle de leur pays, architectures qui ont en commun l'emploi d'une structure poteau poutre et le refus de l'ajout d'ornementation. Même durant la Guerre du Pacifique, les plans architecturaux de Le Corbusier et le design de mobilier introduit par sa collaboratrice Charlotte Perriand représentèrent la "symbiose de la modernité et de la tradition ». Le Japon est parvenu au deuxième rang parmi les pays libres en 1965 quand Le Corbusier disparaît. Celui-ci sera redécouvert au moment où la question de la qualité de la ville se posera.

3 Dans son introduction destinée aux lecteurs français, Gérard Monnier, auteur de Le Corbusier (La Manufacture, 1996), insiste d'abord sur les rapports exceptionnellement riches entre Le Corbusier et le Japon, les travaux de l'architecte-urbaniste s'inscrivant depuis les années 1920 dans l'histoire de l'architecture du pays. Tout en résumant les dix contributions successives, Monnier retrace les démarches des architectes, des urbanistes et des designers nippons qui se sont inspirés de Le Corbusier. Nous comprenons que Le Corbusier était une référence qui permettait de synthétiser la nouveauté et la tradition, l'industrialisation et la création artistique, l'architecture moderniste et la construction d'un Japon moderne.

Dans sa conférence intitulée «Le syndrome Le Corbusier : sur le processus d'évolution de l'architecture moderne au Japon», Maki Fumihiko, architecte, lauréat du Prix Pritzker, compare l'influence corbuséenne à « l'ombre projetée d'un nuage » et la divise en trois phases. La première couvre la fin des années 1920 jusqu'aux années 1930 où Le Corbusier fut perçu comme un jeune héros. La seconde correspond à une période incluant la Guerre du Pacifique et l'après-guerre, autrement dit, avant la haute croissance économique du Japon qui débuta dans la première moitié des années 1960. L'image de Le Corbusier se transforma alors en celle de maître du Modernisme. Lors de la troisième phase, après sa mort, on commence à relativiser et débattre à nouveau de l'ensemble de ses travaux. Maki rappelle enfin, que pour les architectes japonais qui recherchaient un espace approprié à leur époque, c'est Le Corbusier «mieux que quiconque » qui a su exprimer « l'existence d'un espace séduisant ».

Afin de mettre en évidence la diversité des influences corbuséennes, le symposium a été organisé en trois sessions reproduites dans les trois parties de l'ouvrage. La première, intitulée "Le Corbusier, le design et le Japon ", est consacrée à la réception de Le Corbusier dans le monde des arts décoratifs des années 1930. Kashiwagi Hiroshi, historien du design moderne, explique dans son intervention « Le Corbusier et le design japonais : formation du regard au design moderne» que les designers nippons ont remarqué la proximité formelle entre les objets artisanaux traditionnels du Japon et ceux des designers du Mouvement moderne tels que le Bauhaus, Charlotte Perriand et Le Corbusier. Cette découverte leur a permis de revaloriser le design traditionnel du mobilier et des produits du quotidien. Yasuto Ōta, historien de l'art et conservateur du Musée départemental de Kanagawa, traite des relations entre trois personnalités : «Le Corbusier, Charlotte Perriand et Sakakura Junzō ». Ōta explique que Sakakura, qui avait travaillé dans l'agence de Le Corbusier de 1931 à 1936, a réussi à synthétiser la conception de son maître et les éléments architecturaux de son pays natal, notamment dans son plan du Pavillon du Japon à l'Exposition internationale de Paris (1937). Par ailleurs, lorsque Charlotte Perriand a été invitée par le ministère du Commerce et de 
l'Industrie du Japon en 1940, Sakakura, qui l'avait rencontrée à l'agence parisienne, l'a aidée et lui a donné l'occasion de créer des ustensiles ménagers et du mobilier avec des artisans japonais. A son retour en France, Perriand conçoit un mobilier fusionnant les caractères moderniste et vernaculaire du Japon.

Dans la deuxième partie, «Le Corbusier et les débuts de l'architecture moderne au Japon ", trois historiens de l'architecture tentent de clarifier les diverses perceptions de Le Corbusier. Sasaki Hiroshi se base sur son propre ouvrage L'Admiration au Grand Maître (Kyoshō e no shōkei, 2000) pour dresser un tableau des différents degrés de proximité des architectes japonais avec Le Corbusier et son œuvre depuis les années 1920. Il passe en revue les architectes qui ont soit lu des textes de Le Corbusier, soit écrit sur lui, soit travaillé avec lui, soit visité son agence de la rue de Sèvres, soit été inspirés par les publications («Le Corbusier et les architectes japonais»). Fujioka Hiroyasu, dans « L'influence corbuséenne chez les architectes japonais et l'intérêt pour l'œuvre de Le Corbusier dans le Japon d'avant-guerre », précise ce que les architectes japonais apprennent ou ne comprennent pas chez lui. Vers 1928 l'engouement pour l'architecte-urbaniste était à son apogée car les architectes japonais recherchant un rationalisme fondamental l'avaient trouvé dans les textes de Le Corbusier. De plus, ses tracés évoquaient en même temps la monumentalité et une élégance sobre qui existait dans l'architecture traditionnelle du Japon. Il leur était donc aisé d'appréhender son style. Fujioka rappelle cependant que les architectes de l'époque ne s'intéressaient ni à l'esthétique de la machine à appliquer à l'architecture ni à l'urbanisme proposé par Le Corbusier. William J. R. Curtis («De l'imitation à la transformation critique: l'interprétation des œuvres de Le Corbusier ») souligne que les architectes nippons inspirés par les travaux de Le Corbusier ne les imitèrent pas aveuglement. Dans une situation culturelle complexe, afin de répondre à des programmes modernes, ils les interprétèrent, les assimilèrent, les transformèrent. Il cite en exemple Maekawa Kunio et Andō Tadao qui ont permis la cristallisation de certaines idées corbuséennes.

7 Dans la troisième partie, quatre contributeurs s'expriment sur «Le Corbusier et l'architecture japonaise d'après-guerre ». L'architecte Fujiki Tadayoshi qui a participé à la construction du Musée d'Art occidental à Tōkyō raconte le processus et l'arrière-plan de ce projet bilatéral dans «Un réceptacle d'œuvres d'art conçu par un cubiste: le musée national d'Art occidental à Tōkyō, ou Le Corbusier et ses disciples japonais ». La vision globale de Curtis est complétée par la contribution «Une question de filiation: de Le Corbusier à Antonin Raymond et à Tange Kenzō » de Fujimori Terunobu. Ce titre n'indique pas une filiation directe du maître au disciple, mais une filiation entre conceptions architecturales, puisque Raymond et Tange n'ont jamais travaillé avec Le Corbusier. Cependant, Fujimori explique que c'est Raymond qui a réalisé au Japon pour la première fois une architecture corbuséenne avec le projet de sa propre villa (1933). Puis, Maekawa Kunio qui avait été stagiaire à l'atelier de la rue de Sèvres est entré à son retour dans l'agence de Raymond (1930). En 1935, Maekawa ouvrit sa propre agence, et Tange y entra trois ans plus tard à sa sortie de l'Université impériale de Tōkyō. Le jeune Tange s'est fait connaître au niveau national et international par trois études : le Centre commémoratif de la Grande Asie (1941), le Centre culturel japonais à Bangkok (1942), le Mémorial de la Paix à Hiroshima (1949). Fujimori relève dans ces trois projets l'influence remarquable du plan du Palais des Soviets de Le Corbusier. Il résume l'avantage de Tange en trois points : l'architectonique de la réalisation en béton brut de 
décoffrage, la disposition de plusieurs bâtiments dans une mise en scène commémorative et le développement à l'échelle urbaine d'un site organisé.

Les deux dernières observations sur la situation après les années 1960 montrent une distance à l'égard de Le Corbusier. Isozaki Arata, architecte et critique d'architecture, l'affirme dans son intervention «1960, l'année où l'on commence, au Japon, à oublier Le Corbusier ». Tout en analysant plusieurs projets architecturaux ou urbains où l'empreinte de Le Corbusier peut être décelée, Isozaki présente ses propres recherches ainsi qu'un essai sur le monastère de La Tourette. Okabe Noriaki, architecte qui a travaillé à l'agence de Renzo Piano (RPBW), quant à lui, apprécie la mise en scène corbuséenne de l'espace qui permet de réaliser «d'une part un monde constitué par l'intérieur concentré et d'autre part, un monde opposé à l'extérieur " "Réflexion autour du cabanon sur la Méditerranée »). Ce procédé est un caractère spécifique de l'architecte qui s'est beaucoup intéressé à l'architecture de la région méditerranéenne mais pas au bâtiment japonais qui au contraire, d'après Okabe, semble pénétrer dans la nature. Bien que la technique et les matériaux de construction aient changé, la conception spatiale de Le Corbusier a été introduite dans les œuvres architecturales contemporaines. Ainsi, nous comprenons qu'au Japon la figure du maître d'avant-garde s'estompe mais que sa conception spatiale vit toujours.

Les deux Le Corbusier et le Japon ne sont pas édités avec le même objectif. Dans la version française, afin de mettre en avant le raisonnement de chaque contribution, sont supprimées la plupart des notes documentaires relatives aux noms des architectes concernés et à leurs travaux. Quelques passages difficiles à suivre, la citation d'un article de Tange Kenzō par exemple, ne sont pas traduits mais résumés. Dans les annexes, un document inédit est présenté : la « Note de Le Corbusier sur le projet de Musée, le 10 juillet 1956 », qui devait être la lettre destinée à l'ambassadeur du Japon en France lors de la présentation de premières épures du musée national d'Art occidental à Tōkyō.

Les couvertures de chacun des livres sont significatives, car elles semblent représenter l'intérêt des lecteurs de chaque pays. Sur l'ouvrage japonais, un portrait de Le Corbusier pris par son photographe préféré Lucien Hervé, occupe toute la couverture ; pour l'édition française, une photographie de la façade du Musée national d'Art occidental est placée en son milieu. Ainsi dans l'ouvrage originel, la filiation japonaise du maître est accentuée. Il y a une raison : sur ce territoire où l'on est habitué à la disparition des bâtiments, ce n'est pas l'œuvre construite mais la filiation des architectes qui donne confiance aux clients pour leurs projets. Le nom du « maître » qui dirige l'agence est aussi respecté que celui du styliste d'une maison de haute couture. L'édition française se focalise sur les traces de l'architecte dans l'histoire japonaise, entre autres le musée national d'Art occidental.

La raison pour laquelle le gouvernement nippon décida de commander à Le Corbusier le projet du Musée d'Art occidental est notable. Comme Fujiki Tadayoshi l'a raconté dans son intervention, après la défaite du Japon, une collection d'œuvres d'art occidentales fut saisie par les Alliés. Il s'agissait de la collection de peintures impressionnistes et de sculptures de Rodin qui appartenait au millionnaire Matsukata Kōjirō. En 1953, suite à l'application du Traité de paix, le gouvernement français, qui avait la propriété des œuvres d'art en question, en a transféré les droits à l'État japonais à condition que celui-ci construise un musée pour abriter la collection nommée dorénavant Matsukata korekushon (Collection Matsukata). Afin de le réaliser, les autorités des deux pays 
s'accordèrent pour choisir Le Corbusier. Après un court voyage au Japon, l'architecte français dessina plusieurs plans et les confia à ses anciens stagiaires, Maekawa Kunio, Sakakura Junzō et Yoshizaka Takamasa. Après de nombreuses discussions avec leur « maître ", ils édifièrent le bâtiment prototype du musée national d'Art occidental en 1959. Même après sa rénovation, le bâtiment est toujours reconnu comme la seule œuvre architecturale de Le Corbusier au Japon.

En 2007, La Fondation Le Corbusier a ajouté ce musée, avec le plan d'urbanisme de Chandigarh en Inde, aux œuvres à inscrire sur la liste du patrimoine mondial de l'Unesco. Le moment est donc propice à la parution de l'édition française de Le Corbusier et le Japon. L'édifice de Tōkyō et l'ouvrage qui est consacré à l'architecte permettront de réévaluer la vie et l'œuvre du concepteur.

Depuis le symposium Le Corbusier et le Japon, la perception de l'architecte a considérablement évolué. Selon le découpage de Maki, nous en serions à la quatrième phase de son influence. Les Japonais du XxI ${ }^{\mathrm{e}}$ siècle commencent à prendre conscience de leur besoin d'équilibre de vie et deviennent attentifs au confort de leurs logements. Une réflexion autour de l'art de vivre permet de découvrir une nouvelle facette de l'architecte. Le Corbusier n'est plus un agitateur qui proclame la maison machine à habiter. À l'occasion du $120^{\mathrm{e}}$ anniversaire de sa naissance, les médias japonais présentent au grand public l'image d'un créateur complet: architecte, urbaniste, concepteur, peintre, plasticien, éditeur, styliste. Parmi de nombreuses manifestations, l'exposition qui s'est tenue au Musée d'art de Mori est représentative. Intitulée «Le Corbusier Art and Architecture - A Life of Creativity ", elle présente dans la grande salle du $53^{\mathrm{e}}$ étage, près de 400 objets dont trois répliques grandeur nature de logements meublés. On peut «visiter» son atelier de peinture de l'appartement de la rue Nungesser et Coli, un appartement en duplex de l'Unité d'habitation de Marseille, le Cabanon de Roquebrune-Cap-Martin. Ainsi, les visiteurs appréhendent sa conception de l'habitat (d'un prototype de l'habitation de petite taille via le logement-office dit soHo à l'immeuble collectif) et aussi sa vie complète en tant qu'homme social, familial et créatif.

Dans le Japon actuel, le nom de Le Corbusier fonctionne aussi efficacement que celui d'une grande marque dans tous les domaines du design moderne. Autrement dit, les compétences de l'architecte et de l'ingénieur peuvent s'élargir: ceux-ci pourront aborder le design de mobilier, collaborer avec des plasticiens, et donc devenir créateurs. Est-ce une autre version du "Syndrome Le Corbusier» ou une nouvelle perception de la mission de l'architecte? 